\title{
The New Normal of ENT OPD: Adapting Safe Practices
}

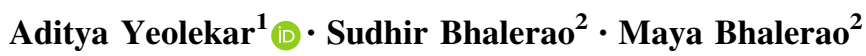

Received: 4 August 2020/Accepted: 2 September 2020/Published online: 14 September 2020

(C) Association of Otolaryngologists of India 2020

\begin{abstract}
The COVID-19 epidemic originating in China has spread rapidly worldwide and converted to pandemic proportions in March 2020. In India and densely populated countries like Brazil and USA the numbers are still rising. Clinicians all over the world are trying to contain it by minimizing the cross-transmission of disease among hospital staff members. In the field of Otorhinolaryngology (ENT) the doctors are exposed to high viral load while examining the patients. Therefore contingency plans are required for dealing with patients in outpatient clinics, and while performing diagnostic endoscopies, minor procedures in OPD and surgeries in operating rooms. Infected patients may shed severe acute respiratory syndrome coronavirus- 2 particles into their environment via body secretions. Therefore, Otolaryngologists should be vigilant. In this submission, we share our experience of an innovative practice plan in redesigning the ENT OPD setup, endoscopy set up and OT so as to reduce the risk of transmission of virus not only to doctors but other healthcare workers. We hope that our modifications will serve as a guide for every Otolaryngologist throughout India towards performing their clinical duties confidently without any apprehension and ensuring adequate safety during this testing times in their small set-up/ clinical establishments.
\end{abstract}

Keywords COVID-19 • Otolaryngology · ENT OPD · The New Normal

Aditya Yeolekar

adidoc@gmail.com

1 Department of ENT and HNS, Smt. Kashibai Navale Medical College, Pune 41, India

2 Bhalerao ENT Hospital, PCMC, Pune, India

\section{Introduction}

The 2019 novel Coronavirus disease (COVID-19) continues to spread rapidly throughout the world in pandemic proportions. The 189th situation report issued by the WHO on July 27, 2020 listed 1, 61, 14, 449 confirmed COVID-19 cases and 6, 46, 641deaths in the world encompassing 213 countries (Weekly Operational Update on COVID-19 9 September 2020_-positive cases: 27,486,960). At the time of preparing this article, although the mortality rate of COVID-19 in India (2.23\%) is not alarming as compared to other counties around the world, the number of positive patients in India have increased to more than 14 lakhs.

Initially it was suggested that animal to human transmission is the mode of spread of the SARS-CoV-2 virus, currently human to human transmission is believed to be the main cause of the spread(Wang and Du 2020) [1]. The virus spreads through minute particles known as aerosols(less than $5 \mu \mathrm{m}$ ) and droplets (more than $20 \mu \mathrm{m}$ ). The spread seems to happen talking out loud, sneezing and coughing in close distances or even distance of $2 \mathrm{~m}-8 \mathrm{~m}$ $[2,3]$.

The aerosolized SARS-CoV-2 particles can remain active in the air possibly three hours or even more [4]. It can stay on surfaces and can spread as fomites. Therefore the contact the hands of the healthcare worker to the contaminated surfaces and retouching them to the face, nose, mouth or hair can potentially infect self [5].

There are reports of doctors succumbing to COVID infection, some of them being ENT surgeons. Such reports have been reported not only in Europe but also in India. ENT Surgeons themselves can contract infection. Consequently, many healthcare personnel have to quarantine themselves due to close contact with the infected otolaryngologists. The most common symptoms of COVID-19 
are fever, dry cough, dyspnoea, myalgia and fatigue [6]. Other symptoms reported include sore throat (5-14\%) and rhinorrhea $(4 \%)[7,9]$. The term asymptomatic carrier is the person who can shed a viral load into even if they have no subtle symptoms and therefore can contribute to spread.[9]. Newer initial presenting symptoms of COVID include anosmia (absent), hyposmia (reduced sense of smell) [9] and dysgeusia (altered taste).

With this in mind we conceptualized, discussed and finalised an innovative practice plan and put it into action. This comprised of redesigning the floor plan of ENT consulting setup, Endoscopy suit and modified the Operation theatre and other logistics required for safe practices at Bhalerao ENT Hospital, PCMC, Pune. We hope that our model will serve as an eye opener for Otolaryngologists and physicians in India and in other relevant clinical specialties who work in close contact with patients like dentists, cosmetic surgeons and ophthalmologists. This will enable the doctors specially ENT Surgeons to carry out their clinical duties safely and effectively. This model will be in practice as, "The New Normal", either till the disease load subsides or the down-slope of the pandemic graph is reached, the timeframe of which is as yet indeterminate.

\section{Modifications in ENT Outpatient Department}

\section{Appointments}

Since June 2020, we streamlined the flow of patients to minimize the risk of cross-transmission. Specifically the patients suffering from head and neck cancers, airway emergencies, head and neck abscesses, acute otitis media, acute mastoiditis, complicated sinusitis, sudden hearing loss, facial paralysis or facial trauma and vertigo are given priority. Pediatric and pregnant patients are always given priority over other patients. The appointments are spaced at adequate intervals. The waiting room is redefined with spacing between two seats. Till the time first patient is out of the complex, next patient is preferably instructed to wait outside the clinic or in their cars. Walk in patients are discouraged.

\section{Entrance}

Only the patient is allowed to go inside while the relative waited outside the clinic. A sanitizer and surgical mask box is kept at the entry of the clinic facility. A acrylic partition ( $3 \mathrm{~mm}$ thick) is installed to serve as the first physical barrier between the healthcare worker and the patients. Patients are triaged at clinic and asked about fever, travel history, occupational exposure, contact history and symptoms and full contact details and address is noted. At the entrance the attendant ask for symptoms and records Heart Rate, SpO2, Temperature by Handheld scanner.

Those not fitting into normal parameters are referred to flu clinics or designated hospitals according to the local government guidelines. Arrows are marked on the floor for directions to the examination room.

\section{Reception Area}

The reception area is separated from clinics and patient area, by an imported acrylic $(3 \mathrm{~mm})$ barricade that are from 5 foot above the reception counter with a window of size one feet by one and a half feet with a plastic flap on the reception side to keep it closed when not required.

\section{ENT OPD Room}

The ENT consultant and the patient occupy separate rooms having a separate entrances to each. Both are separated by a acrylic door of $3 \mathrm{~mm}$ thickness ( 7 feet $\times 3$ feet). There are two airtight circular slots or ports in this door three feet from below with adequate space between two slots. To this are attached yellow thick latex heavy weight gloves of arms length are attached. This model is based on the swab collection kiosks used in the state of Kerala as shown in Fig. 1.

A nurse/ healthcare worker wearing full level II PPE helps the ENT surgeon for entire ENT examination and ear suction procedures in the patients room (Fig. 2).

After examination of the patient the gloves are cleaned with solution of Sodium Hypochlorite.

All examination details with prescription are entered into computer software and delivered to patient at the exit. None of the rooms have air conditioners. Adequate cross ventilation is ensured and exhaust rooms fitted in all rooms.

\section{Endoscopy Box}

An acrylic endoscopy chamber of size 3 feet $\times 2.5$ feet $\times$ 2 feet is used to cover the patient completely while performing nasal endoscopy. It has 4 air tight ports through which high quality elbow length latex heavy weight gloves can pass inside. Two are for the surgeon and two for the assistant. This is covered by sterile plastic drape unto the feet of the patient. (Fig. 3)This barrier helps to prevents spread droplets and aerosols if any generated during the procedure. This is also helpful for dressing of surgical wounds and suture removal.

\section{Endoscopy Room}

Nasal endoscopy flexible and 90 degree laryngoscopy can induce coughing, sneezing and choking and hence 


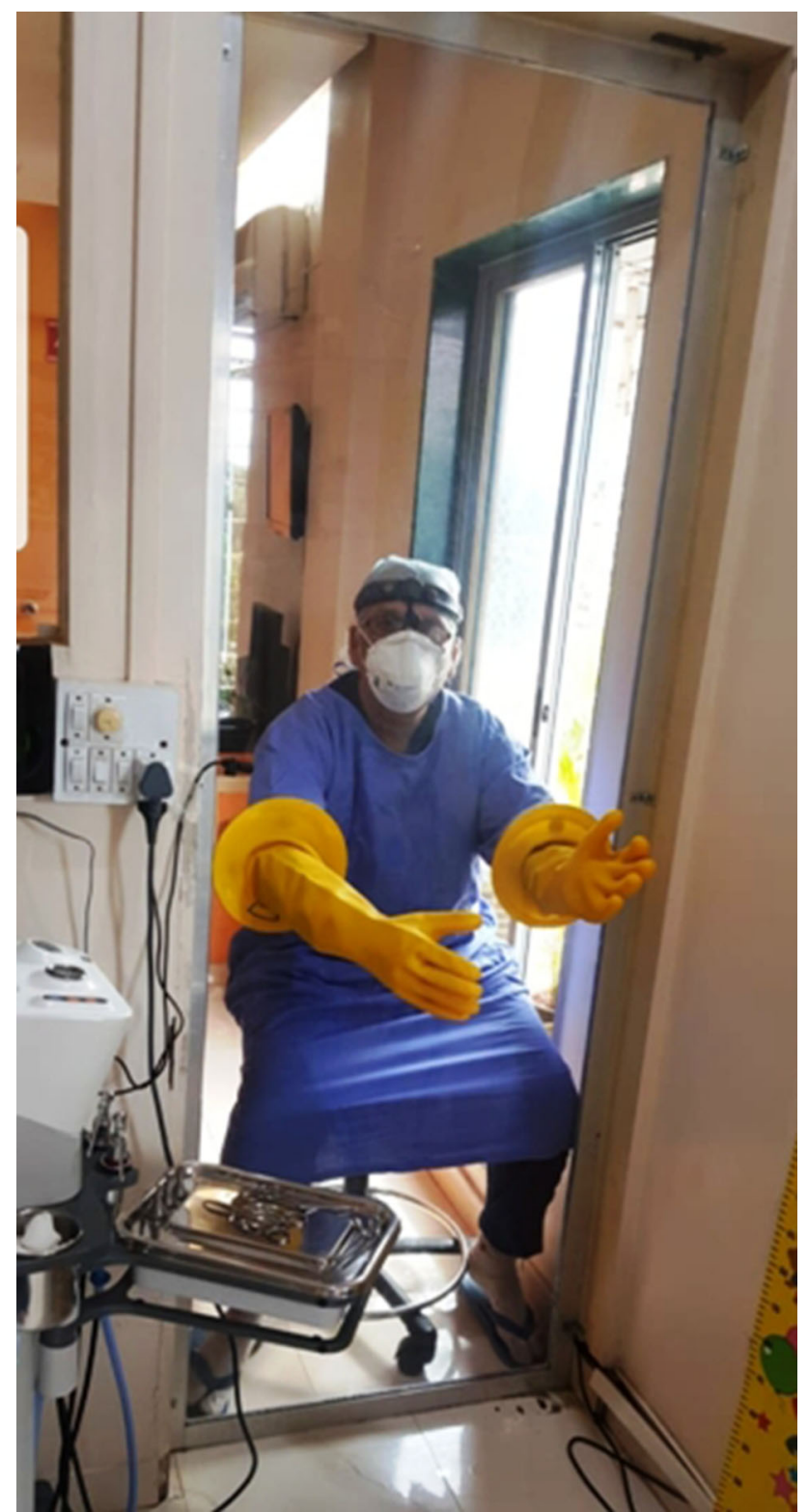

Fig. 1 Full length acrylic door between doctor room and patient room with two airtight glove arms

potentially droplet generating procedures. These were performed in a separate designated endoscopy room (Fig. 4). Additionally, patients were asked to wear a surgical mask at all times. In the clinic, the doctors and healthcare assistant performing procedures wear protective equipment (level II PPE) (e.g., face shield, N95 respirator, long-sleeved disposable fluid-resistant gown and disposable gloves), according the guidelines issued by the Government of India. (https://www.mohfw.gov.in/pdf/ ENTCOVID0306.pdf).

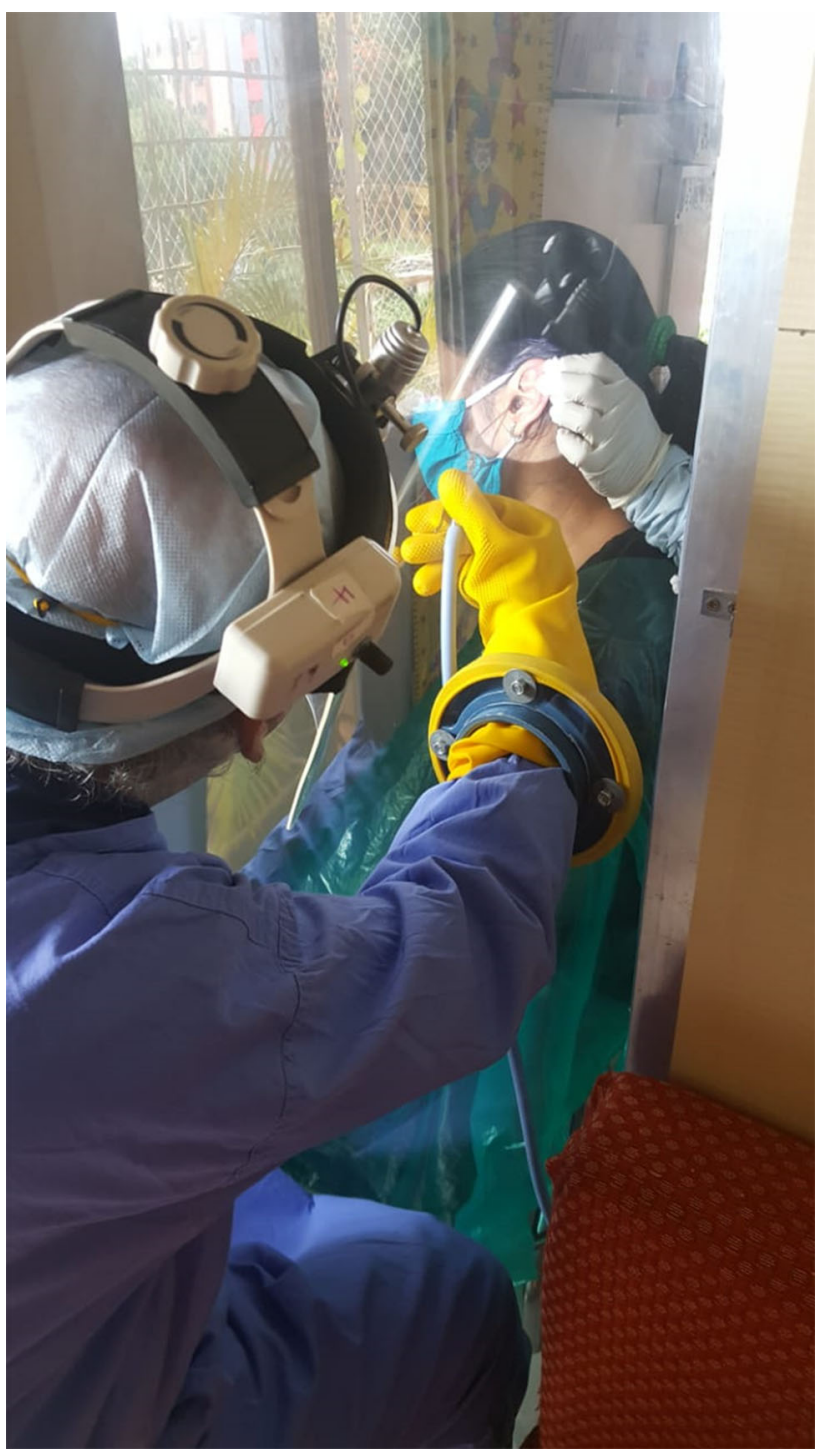

Fig. 2 Minor procedures and ear suction being done through the partition

\section{Operation Theatre}

The operation theatre is operated according to the guidelines issued by the Govt. of India.

Additionally like an endoscopy box, an acrylic box for OT is made of dimensions 4 feet $\times 3$ feet $\times 2$ feet closed from three sides with windows in them is used (Fig. 5). The height of three feet allows enough space for movement of surgical hand and handling of instruments. This is covered by a plastic drape up to the feet of the patient.

This acrylic box is open on the sides just to park the head of the microscope.

The microscope and the acrylic box are covered all around with a plastic impermeable sheet, to prevent aerosol and droplet spread while drilling (Fig. 7). 


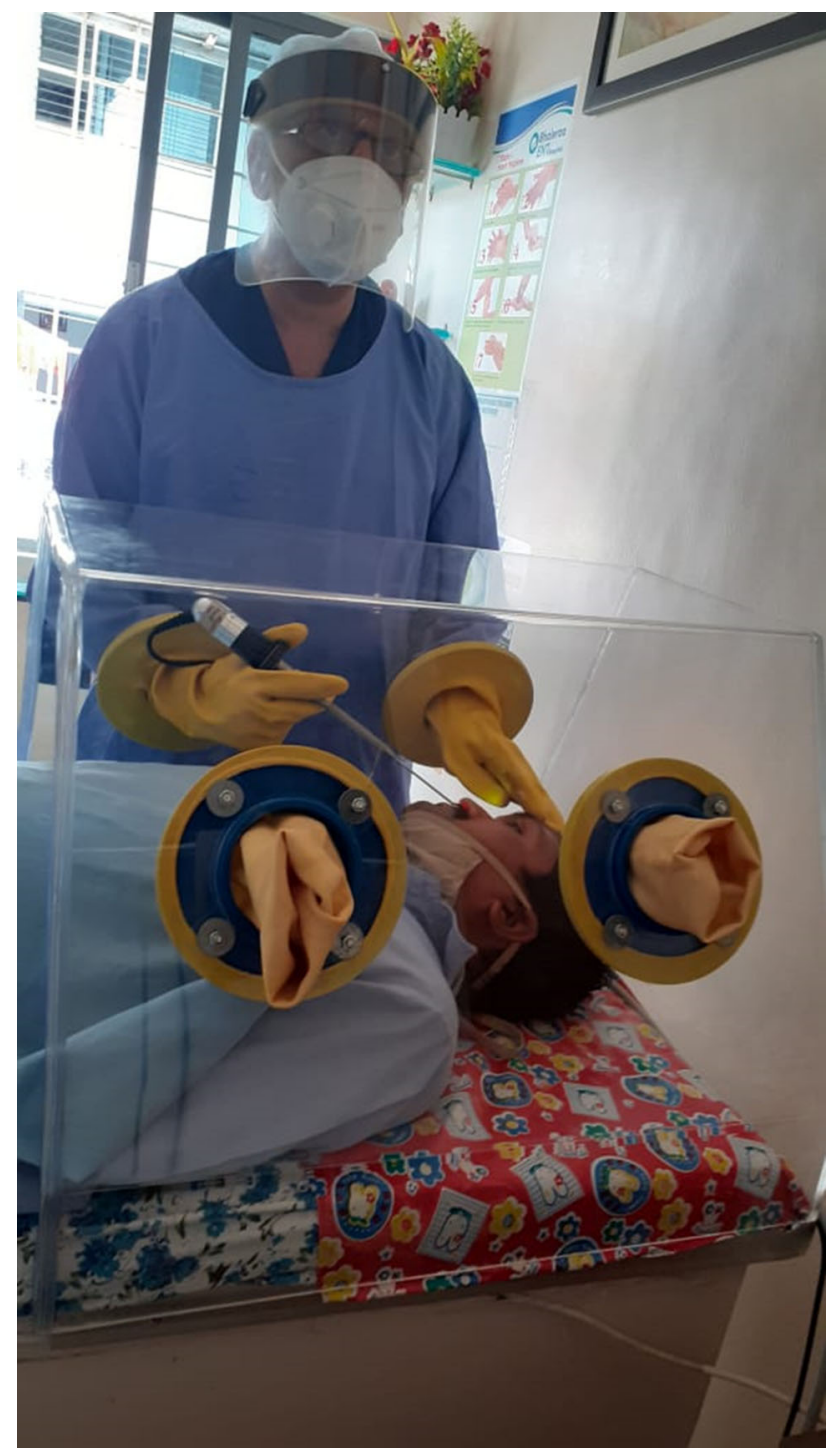

Fig. 3 Endoscopy Box

All operative procedures and instrument handling are done under the plastic sheet mount to prevent any spread of possible infection. The bone dust and blood stains soil the inner lining of the plastic drape that is disposable, thereby ensuring safety of the healthcare staff. In this thick plastic sheet $\mathrm{U}$ shaped flaps are made for entry and exit of hands like a flap. This ensures a complete no touch technique.

\section{Anesthesia Considerations}

Local Anaesthetia is preferred to reduce aerosol generation and to reduce patient risk from during intubation and extubation of general anaesthesia. It is recommended that extubation is done in the operating theatre smoothly without any coughing. All surgeries are carried out in a designated COVID-19 free area wherever practical to avoid exposure to potentially COVID-19 positive areas of

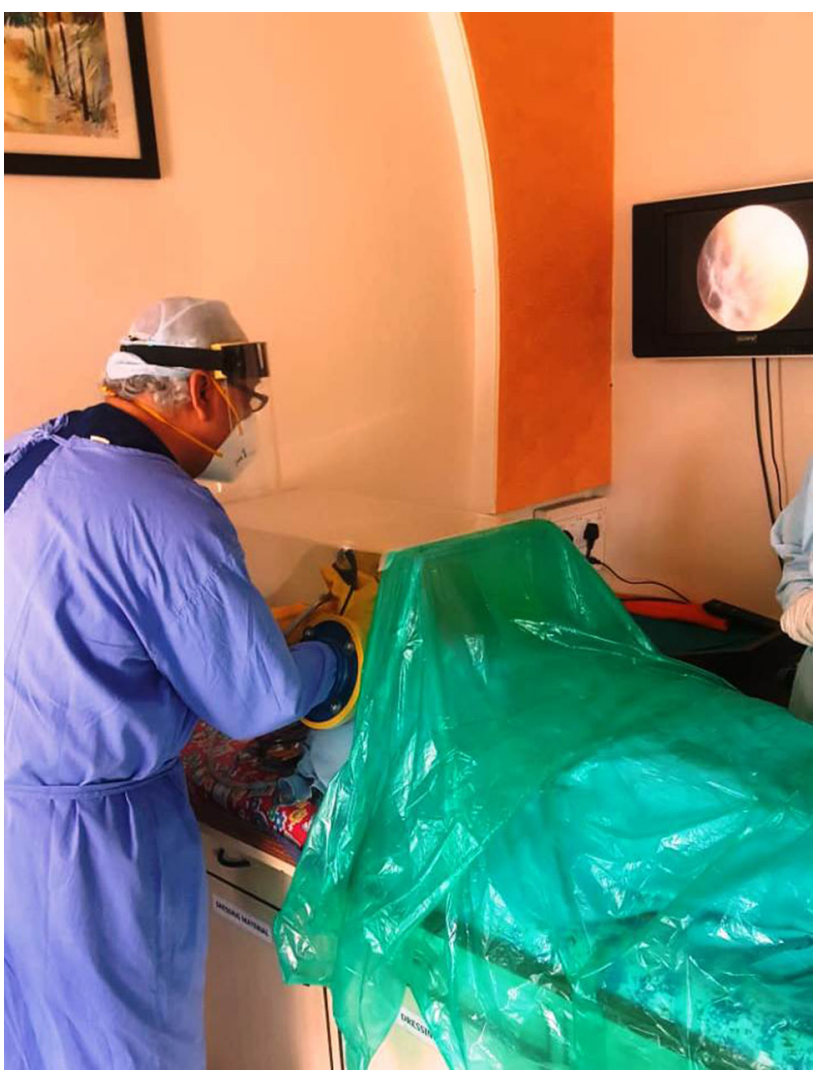

Fig. 4 Diagnostic Nasal Endoscopy using a box

the hospital. These surgeries are undertaken as daycare procedures wherever possible. Elective surgeries are planned with prior antigen testing of patient by RT-PCR to be found negative $48 \mathrm{~h}$ prior to surgery. PPE are removed and disposed off outside the OT in dedicated doffing areas.

\section{Cleaning and Disinfection}

(As mentioned in guidelines issued by Govt. of India).

Freshly prepared $1 \%$ Sodium Hypochlorite is used as a disinfectant for cleaning and disinfection.A contact time of at least $10 \mathrm{~min}$ is followed. All clinical set up cleaning is done with detergent with water alternately with $1 \%$ Sodium Hypochlorite. High contact surfaces (door and door knobs) are cleaned with $1 \%$ Sodium Hypochlorite every half hourly basis. Nursing station, telephones and computers are cleaned regularly. Alcohol (e.g. isopropyl $70 \%$ or ethyl alcohol $70 \%$ ) is used to wipe down metallic surfaces. BP apparatus, thermometers and endoscopes are cleaned with $70 \%$ alcohol-based rub/spirit swab before each patient. Endoscopes are sterilised by immersing in $2 \%$ glutaraldehyde solution for $20 \mathrm{~min}$. ENT steel instruments are autoclaved after every use. 

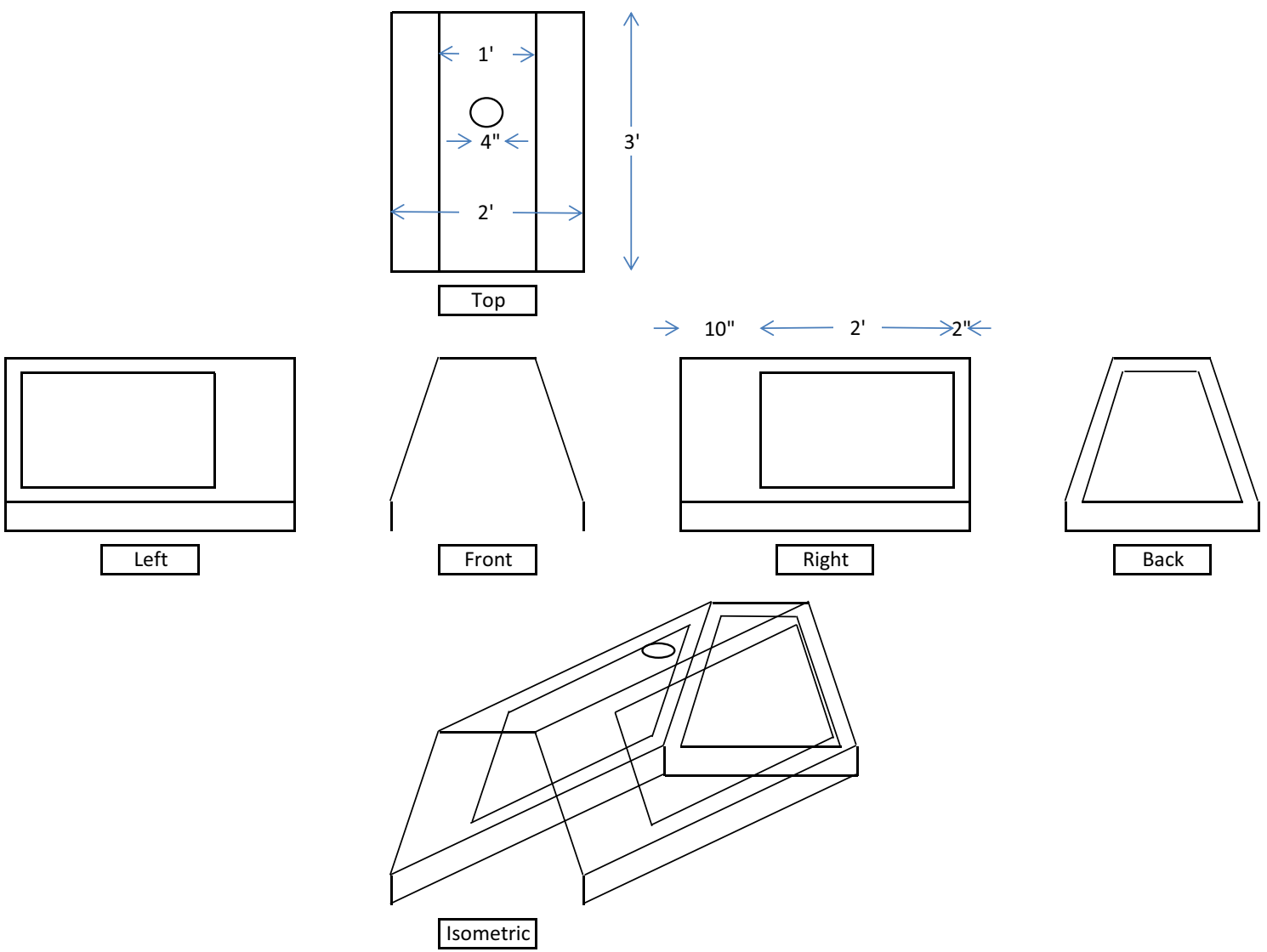

Fig. 5 Dimensions of acrylic OT box.The head end has an opening through which the anesthetist intubates and extubates the patient.(Fig. 6)

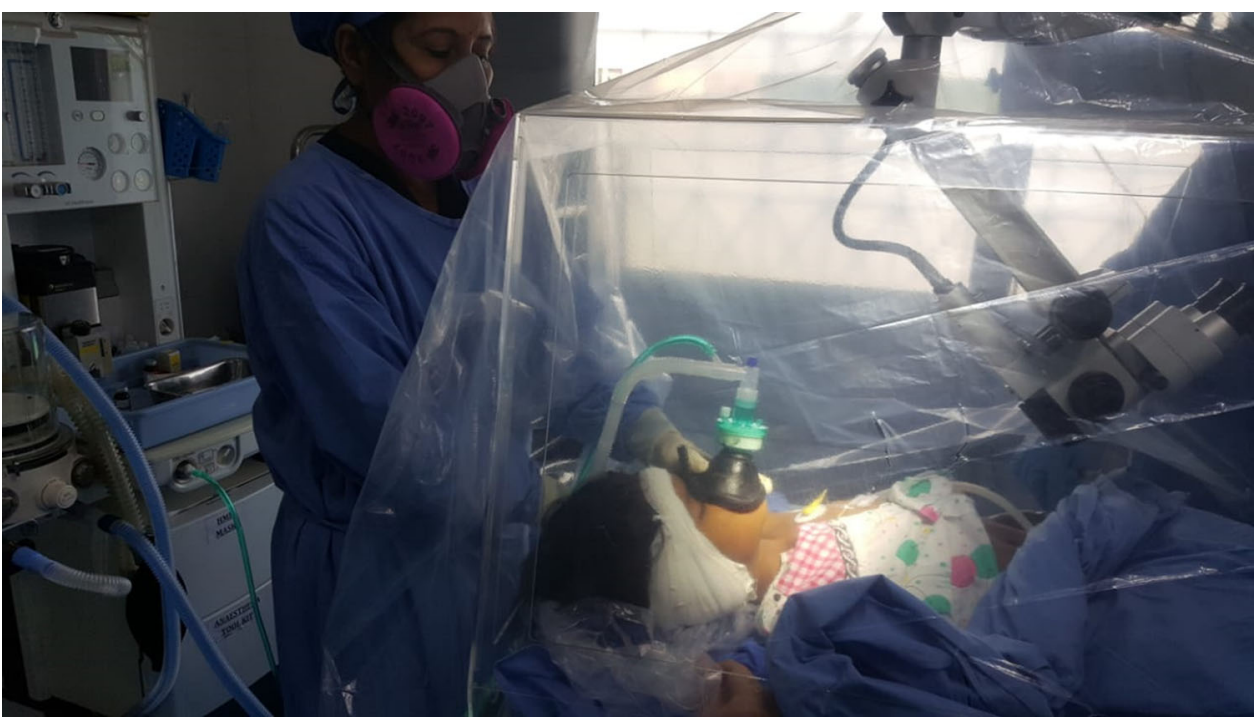

Fig. 6 The Direct Laryngoscopy GA acrylic box

\section{Application of Telemedicine}

One can adopt the strategy of video consultation for empirical treatment based on patient's symptoms. A photo of patients own throat can be asked and shared online via whatsapp to see for any disease in the oropharynx. One can use various private applications available in market. A digitally signed prescription is given to the patient with 
Fig. 7 Otology surgery carried out under the plastic hood mounted on microscope and under the arcylic box

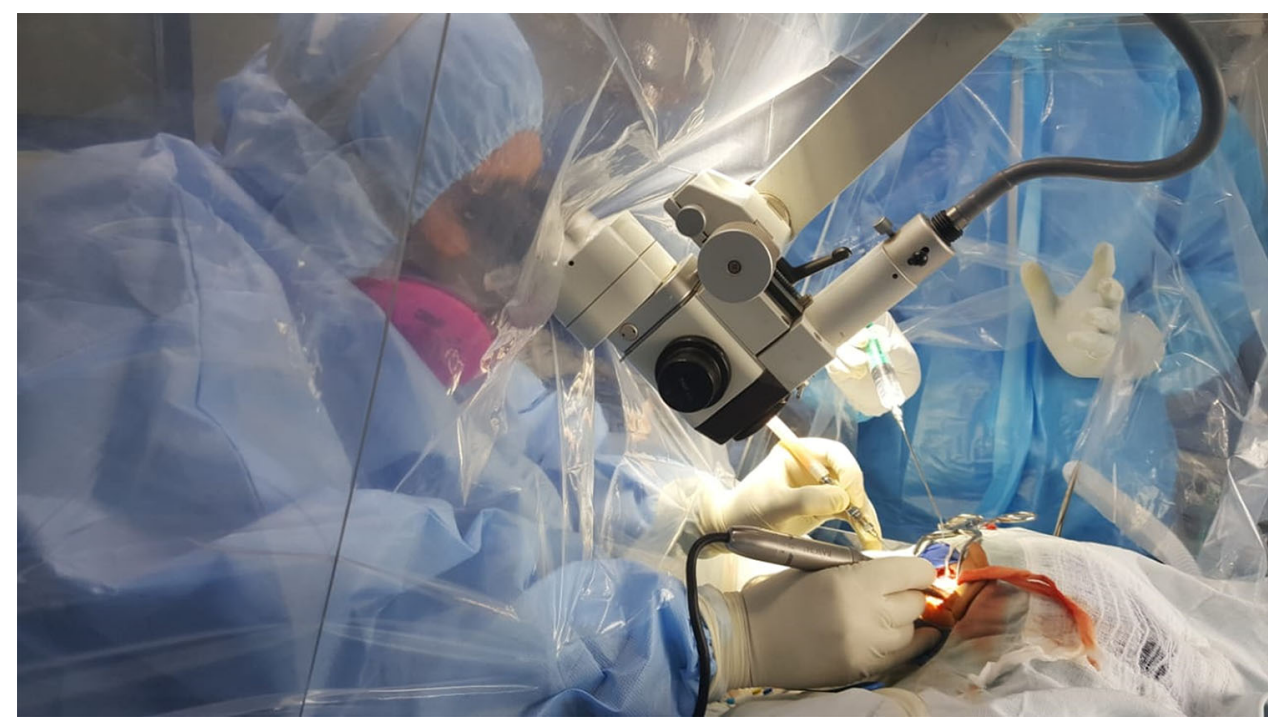

probable diagnosis. A copy of it can be saved for further reference. Teleconsultation also suitable for the followup of patients with nasal symptoms like allergy or hearing impairments. In our opinion pediatric and pregnant women and those with exclusive ear complaints should be consulted in person. This helps in precise diagnosis of otomycosis, acute suppurative otitis media and the more dangerous cholesteatoma.

Also a patient suffering from vertigo and balance disorders should be examined in person to pinpoint the diagnosis and offer complete treatment.

\section{Health Education}

Health education in healthcare personnel plays a very important role in preventing cross infection among the staff, patients visiting clinical OPD's and their family members. This shall include topics on the structure of virus, mechanism of spread, clinical features of COVID, treatment and primary prevention strategies. Many such self learning informative videos are available on the websites of Govt. of India, Ministry of Health and Family Welfare (training material) https://www.mohfw.gov.in/, WHO -https://openwho.org/channels/covid-19 and Maharashtra University of Health Sciences (MUHS). Other important aspects that need to be included are proper concepts of infection control like hand hygiene, the donning and doffing of personal protective equipment (PPE), the handling of suspected or confirmed cases of COVID19 , the proper disposal of clinical waste and the handling of instruments and laboratory samples.

\section{Results}

This innovative practice was put into action from 16 June 2020 and is continued thereafter.Within the forty day period 16 June to 25 July 2020, 1279 patients were examined. Out of these 10 patients suspected to have COVID were tested and 4 patients were detected to be positive and were guided to dedicated COVID centre. None of the 5 doctors and 20 healthcare workers working in the hospital had any symptoms of flu like illness during this period and they continue to function safely. This is an indirect validation of our innovation.

\section{Discussion}

COVID-19 is a pandemic disease worldwide, and to date about 1, 61, 14, 449 have been infected by SARS-CoV-2 virus. Since ENT Surgeons are exposed to nose and oral cavity while examining patients which is the very entry and exit portal of this virus, it is imperative upon the doctors and healthcare workers to be on vigil and adopt safe practices. The ways and means to tackle spread include following social distancing norms, wearing of masks, avoid overcrowding and wearing of PPE whenever applicable. Certain other modifications are required to be done at an individual ENT OPD/Operative setup to prevent further possible transmission. This innovative modification might help ENT surgeons across India to work with confidence in their clinical establishments. This further can be adopted by other medical specialties like dentistry and cosmetic surgery. 


\section{Conclusion}

To sum up, till the time the vaccine is not established to provide adequate antibody titres and confer lasting immunity, the feeling of uncertainty and apprehension is expected to prevail for next few months. The course of COVID-19 pandemic continues to be indeterminate. Therefore a time has come to embrace, "The New Normal", by such innovative practices that prove safe. It will help every doctor to work in a well protected environment without any fear.

Funding None.

\section{Compliance with Ethical Standards}

Conflict of interest The authors declare that they have no conflicts of interests.

Ethical Approval All the research included in this manuscript meet the ethical guidelines, including adherence to the legal requirements of the study country. The study protocol complied with the 1964 Helsinki declaration and its later amendments.

Informed Consent Not applicable.

\section{References}

1. World Health Organization (WHO) (2004). Technical Report, Series No. 924. https://www.who.int/biologicals/technical_ report_series/en/ [Google Scholar].

2. Bourouiba L (2020) Turbulent gas clouds and respiratory pathogen emissions: potential implications for reducing transmission of COVID-19. JAMA. https://doi.org/10.1001/jama. 2020.4756
3. Loh NHW, Tan Y, Taculod J, Gorospe B, Teope AS, Somani J, Tan AYH (2020) The impact of high-flow nasal cannula (HFNC) on coughing distance: implications on its use during the novel coronavirus disease outbreak. Can J Anesth. https://doi.org/ 10.1007/s12630-020-01634-3

4. Kligerman MP, Vukkadala N, Tsang RKY, Sunwoo JB, Holsinger FC, Chan JYK et al (2020) Managing head and neck cancer patients with tracheostomy or laryngectomy during the COVID-19 pandemic. Head Neck. https://doi.org/10.1002/ hed.26171

5. Gengler I, Wang JC, Speth MM, Sedaghat AR (2020) Sinonasal pathophysiology of SARS-CoV-2 and COVID-19: a systematic review of the current evidence. Laryng Investig Otolaryngol. https://doi.org/10.1002/lio2.384

6. Huang C, Wang Y, Li A et al (2020) Clinical features of patients infected with 2019 novel coronavirus in Wuhan. China Lancet 395:497-506

7. Chen N, Zhou M, Dang X et al (2020) Epidemiological and clinical characteristics of 99 cases of 2019 novel coronavirus pneumonia in Wuhan, China: a descriptive study. Lancet 395:507-513

8. Yang W, Cao Q, Qin L et al (2020) Clinical characteristics and imaging manifestations of the novel 2019 coronavirus disease (COVID-19): a multi-center study in Wenzhou city, Zhejiang, China. J Infect. https://doi.org/0.1016/j.jinf.2020.02.016

9. Wang Y, Liu Y, Liu L et al (2020) Clinical outcome of 55 asymptomatic cases at the time of hospital admission infected with SARS - coronavirus in Shenzhen. China. J Infect Dis. https://doi.org/10.1093/infdis/jiaa119

10. Lai CC, Liu YH, Wang CY et al (2020) Asymptomatic carrier state, acute respiratory disease, and pneumonia due to severe acute respiratory syndrome coronavirus 2 (SARS-CoV-2): fact and myth. J Microbiol Immunol Infect. https://doi.org/10.1016/ j.jmil.2020.02.012

11. Heidari F, Karimi E, Firouzifar M et al (2020) Anosmia as a prominent symptom of COVID-19 infection. Rhinology 58(3):302-330. https://doi.org/10.4193/Rhin20.140

Publisher's Note Springer Nature remains neutral with regard to jurisdictional claims in published maps and institutional affiliations. 